\title{
VARs, common factors and the empirical validation of equilibrium business cycle models
}

\author{
Domenico Giannone \\ ECARES, Université Libre de Bruxelles \\ Lucrezia REICHLIN \\ ECARES, Université Libre de Bruxelles and CEPR \\ Luca SALA \\ ECARES, Université Libre de Bruxelles \\ IGIER - Universitá Bocconi
}

December 9, 2002

\begin{abstract}
Equilibrium business cycle models have typically less shocks than variables. As pointed out by Altug, 1989 and Sargent, 1989, if variables are measured with error, this characteristic implies that the model solution for measured variables has a factor structure. This paper compares estimation performance for the impulse response coefficients based on a VAR approximation to this class of models and an estimation method that explicitely takes into account the restrictions implied by the factor structure. Bias and mean squared error for both factor based and VAR based estimates of impulse response functions are quantified using, as data generating process, a calibrated standard equilibrium business cycle model. We show that, at short horizons, VAR estimates of impulse response functions are less accurate than factor estimates while the two methods perform similarly at medium and long run horizons.
\end{abstract}

JEL subject classification : E32, C33, C52

Key words and phrases : Dynamic factor models, structural VARs, identification, equilibrium business cycle models. 


\section{Introduction}

The basic econometric tool for empirical validation of macroeconomic models is the Vector Autoregressive Model (VAR). This model is easy to estimate and, once identification restrictions are imposed, it can be used to evaluate the impact of economic shocks on key variables.

In structural VAR macroeconomics, variables are represented as driven by serially uncorrelated shocks, each having a different source or nature, like "demand", "supply", "technology", "monetary policy" and so on. Each variable reacts to a particular shock with a specific sign, intensity and lag structure, summarized by the so called "impulseresponse function". Implications of economic theory not used for identification can then be compared with estimation results and tested.

A strong motivation for the use of VARs is that stochastic general equilibrium macroeconomic models have solution that can be represented in VAR form and therefore VAR econometrics provide the tool to bridge theory and data.

The typical theoretical macro model, however, has few shocks driving the key variables in the macroeconomy. In the first generation real business cycle models, for example, one shock - technology - is responsible for volatility of output, consumption and investment both in the short and long-run. In that stylized economy, there is only one source of variation. Other models take into account shocks in preferences or money, but sources of macro variations remain few.

The implication of this feature is that equilibrium business cycle models have reduced stochastic rank (i.e. the spectral density of the observation has reduced rank). A further implication, as observed by Altug, 1989 and Sargent, 1989, is that, when variables are measured with errors, the model for measured variables has a dynamic factor analytic structure. Since it can be easily shown that, with measurement error, the reduced form solution follows a VARMA model, from the estimation point of view, there are two approximations to the measured model that one my consider: a VAR model with a sufficiently large number of lags or a method that takes explicitely into account the restrictions implied by the model (factor model estimation).

Dynamic factor models imply a restriction on the spectral density of the observations whereby the latter can be expressed as the sum of two orthogonal components, the spectral density of the common component, of reduced rank, and the spectral density of the idiosyncratic component, of full rank. The former captures all the covariances of the observations at leads and lags while the latter is diagonal and can therefore represent non cross-correlated measurement error. The factor literature, which has wide applications in many fields other than economics, has been first introduced in macroeconomics by Sargent and Sims, 1977 and Geweke, 1977 and further developed by Geweke and Singleton, 1981 and Engle and Watson, 1983. Recently, factor models have been rediscovered in macroeconomics as a tool for analysing large panels of time series (Forni and Reichlin, 1998, Forni, Hallin, Lippi and Reichlin, 2000, Stock and Watson, 2002 and related literature). For empirical evidence of stochastic rank reduction, see Altissimo et al, 2002 on European data and Giannone et al., 2002 on US data.

The objective of this paper is to evaluate the performance of these two alternative approximations under different hypotheses on the size of measurement error. We will 
perform this comparison by generating data from a simple business cycle model with and without measurement error and comparing impulse response estimates from VAR and factor procedures. We estimate the loadings of the common factors by a quasimaximum likelihood procedure proposed by Doz and Lenglart, 1999. We show that, since impulse response functions are continuous functions of the loadings, the latter can be estimated consistently (on this point, see also Giannone, Reichlin and Sala, 2002). The intuition of this result is that the factor model helps to clean data from measurement error by exploiting the theoretical (and empirical) feature of stochastic rank reduction.

Measurement errors, we will show, contaminate VAR impulse response functions at all horizons. Under the assumption of poorly autocorrelated measurement error and persistent dynamic in the model economy, the contamination affects contemporaneous and short-term responses in particular, while factor estimates are more precise in the short run and provide a similar degree of precision at all horizons. The latter depends of course on the size of the error. Our results suggest that VAR estimates are more reliable in the medium and long-run than in the short-run. They also explain the empirical finding that while macroeconomic time series display cointegration (i.e. they show reduction of rank of the spectral density of their first differences at zero frequency), it is more difficult to find evidence of rank reduction at higher frequencies: in the longrun, measurement error is smaller and, as a consequence, underlying collinear relations are more evident than at higher frequencies.

The paper is organized as follows. In the first Section, we will describe the general linear solution of equilibrium business cycle models and then illustrate a special simple case. In the second Section, we discuss VAR and factor estimates with and without measurement errors. In the third, we perform the empirical experiment based on the simple model. The last Section, before the conclusions, is a general discussion which relates traditional factor models used in this paper with the more recent literature on factor models for large panels of time series.

\section{A model economy and VAR analysis}

\subsection{Equilibrium business cycle models}

\section{A. General Structure}

Let us recall the general structure of an equilibrium business cycle model. In this framework, as it is well known, the problem in the decentralized economy is the same as the social planner's. The latter maximizes the utility of the representative agent:

$$
\max \mathrm{E}_{0}\left[\sum_{t=0}^{\infty} \beta^{t} U\left(X_{t}, Y_{t}\right)\right]
$$

subject to the feasibility constraints::

$$
f\left(X_{t}, X_{t-1}, \cdots, Y_{t}, Y_{t-1}, \cdots, S_{t}, S_{t-1}, \cdots\right) \leq 0
$$




$$
S_{t}=g\left(\epsilon_{t}, \epsilon_{t-1}, \cdots\right)
$$

where $X_{t}$ is the $m \times 1$ vector of endogenous predetermined variables, $Y_{t}$ is the $n \times 1$ vector of the endogenous non predetermined variables and $S_{t}$ is the $q \times 1$ vector of exogenous variables (the number of variables considered is therefore $N=m+n+q$ ). The parameter $\beta$ defines the discount factor and $\epsilon_{t}$ is a $q$ dimensional i.i.d. normal process with mean 0 and variance $\Sigma_{\epsilon}$.

Stated at this level of generality, the model encompasses several examples in the literature, from the simple real business cycle model á la King, Plosser and Rebelo, 1991, to the time-to-build economy á la Kydland and Prescott, 1983 to the model with heterogenous capital (Campbell, 1997). Indicating with small letters the difference between the log of the variables and their non-stochastic steady state, the solution of such models has the following recursive structure:

$$
\begin{gathered}
\Psi(L) s_{t}=\epsilon_{t} \\
C(L) x_{t}=D(L) s_{t} \\
y_{t}=\Lambda_{1}(L) x_{t}+\Lambda_{2}(L) s_{t}
\end{gathered}
$$

where:

$$
\begin{gathered}
C(L)=C_{0}+C_{1} L+\ldots+C_{p_{c}} L^{p_{c}} \\
D(L)=D_{0}+D_{1} L+\ldots+D_{p_{d}} L^{p_{d}} \\
\Lambda_{1}(L)=\Lambda_{1,1} L+\ldots+\Lambda_{1, p_{\Lambda_{1}}} L^{p_{\Lambda_{1}}} \\
\Lambda_{2}(L)=\Lambda_{2,0}+\Lambda_{2,1} L+\ldots+\Lambda_{2, p_{\Lambda_{2}}} L^{p_{\Lambda_{2}} .}
\end{gathered}
$$

It should be noticed that this solution form applies even to a larger class of models than those based on the maximization problem described above. As Christiano, 2001 has pointed out, more complex models with heterogenous agents and different informations sets, also have the same solution structure. This can be understood by noticing that the length of the filters $\Lambda_{1}(L)$ and $C(L)$ is determined by the lags of predetermined variables necessary for the determination of the endogenous and the predetermined variables while the filters $\Lambda_{2}(L)$ and $D(L)$ accomodate for the possibility that endogenous variables are determined on the basis of different information sets.

Defining the vector of all the observables as $w_{t}=\left[y_{t}^{\prime} x_{t}^{\prime} s_{t}^{\prime}\right]^{\prime}$, the solution, written in its constrained VAR form, is:

$$
A(L) w_{t}=B \epsilon_{t}
$$

where,

$$
A(L)=\left(\begin{array}{lll}
I_{n} & -\Lambda_{1}(L) & -\Lambda_{2}(L) \\
0 & C(L) & -D(L) \\
0 & 0 & \Psi(L)
\end{array}\right)
$$


and:

$$
B=\left(\begin{array}{l}
0_{(n \times q)} \\
0_{(m \times q)} \\
I_{q}
\end{array}\right)
$$

The dynamic rank of this system of equations, defined as the rank of the spectral density matrix of $w_{t}$ is $q$, with $q<N$. The model, therefore, has reduced dynamic rank.

It is also customary to write the solution in its static state space representation where the vector of state variables includes the lagged predetermined variables, and current and lagged exogenous variables. The latter is defined as $F_{t}=\left[x_{t-1}^{\prime} \ldots x_{t-p_{x}}^{\prime} s_{t}^{\prime} \ldots s_{t-p_{s}}^{\prime}\right]^{\prime}$, where $p_{x}=\max \left\{p_{\Lambda_{1}}, p_{c}\right\}$ and $p_{s}=\max \left\{p_{\Lambda_{2}}, p_{d}\right\}$, while the variables in the vector $w_{t}$ are expressed as contemporaneous linear combinations of $F_{t}$ :

$$
w_{t}=\Lambda F_{t}
$$

with:

$$
H(L) F_{t}=K \epsilon_{t}
$$

The dimension of the vector of state variables in this static representation is $r=$ $m p_{x}+q\left(p_{s}+1\right)$ and it therefore depends on the $p_{x}$ and $p_{s}$ lags included in the model as well as on $q$ and $m$. This is also an upper bound for the rank of the contemporaneous variance-covariance matrix of $w_{t}, \Gamma_{w}(0)=\mathrm{E} w^{\prime} w$ and defines the static rank of the system.

Static and dynamic rank reveal different features of the model economies. Reduced dynamic rank $q$ tells us that only $q$ shocks matter for dynamics and therefore is a consequence of the characteristics of the exogenous forces driving the economy, while the static rank depends in general on the structure of the economy (the zero restrictions on the coefficients of the VAR form) and on the number of lags included ${ }^{1}$. Typically, models with rich dynamics, such as, for example, the time-to build model á la Kydland and Prescott, 1983, have reduced stochastic rank but may have full static rank while simpler models have both reduced static and dynamic rank. Static and dynamic ranks must be thought as restrictions, in principle testable, derived from theory. Moreover, rank reduction has implications for estimation that we will develop below.

To clarify the structure of the model and the role of the filters, as well as the role of rank reduction it will be useful to discuss a specific example of the general model. The same example will be used in the empirical Section.

\footnotetext{
${ }^{1} \mathrm{~A}$ different restriction implies rank reduction of the lagged VAR matrices. In this case the solution will have reduced rank representation as in Ahn and Reinsel, 1988, and Velu et al., 1986, or common features as defined in Engle and Kozicki, 1993, and, under further restrictions, common cycles as in Vahid and Engle, 1993
} 


\section{B. The basic business cycle model}

What we illustrate here is a simplified version of King, Plosser and Rebelo, 1991, which is also the textbook example analyzed by Uhlig, 1998, to which we refer for all details.

The model can be seen as a special case of what discussed in A. where there is only one source of variability - technology -, labor is exogenous, there are no time to build features, agents are homogeneous and have the same information set. We have: $n=3$, $m=1, q=1$ and $\Psi(L)=1-\psi L$. The only exogenous state variable is productivity, $z_{t}$, which, with lagged capital stock $k_{t-1}$, form the vector of the state variables. By using a standard functional form for the utility function, we can write the maximization problem as:

$$
\max U=\mathrm{E}_{0}\left[\sum_{t=0}^{\infty} \beta^{t} \frac{C_{t}^{1-\eta}-1}{1-\eta}\right]
$$

subject to:

$$
\begin{aligned}
C_{t}+K_{t} & =Z_{t}+K_{t-1}^{\rho}+(1-\delta) K_{t-1} \\
\log \left(Z_{t}\right) & =\log \bar{Z}+\psi \log \left(Z_{t-1}\right)+\epsilon_{t}
\end{aligned}
$$

where $C_{t}, K_{t}$ define consumption and the capital stock and $Z_{t}$ is the productivity exogenous process. The parameters $\delta, \rho, \eta$ and $\psi$ define, respectively, the discount factor, the depreciation rate, the capital share, the coefficient of relative risk aversion and the autoregressive parameter governing persistence of the technology shock in the productivity equation.

Notice that in this case $p_{\Lambda_{1}}=p_{c}=1, p_{\Lambda_{2}}=p_{d}=0$. We have $y_{t}=\left[r_{t} c_{t} y_{t}\right]^{\prime}$ where $r_{t}$ is the real interest rate and $y_{t}$ is output; moreover, $x_{t}=k_{t}$ and $s_{t}=z_{t}$ (lower cases define, as before, variables in log and deviation from their non stochastic steady state).

The VAR solution can be written as:

$$
A(L) w_{t}=B \epsilon_{t}
$$

where:

$$
A(L)=\left(\begin{array}{ccc}
I_{3} & -\Lambda_{1} L & -\Lambda_{2} \\
0 & (1-C L) & -D \\
0 & 0 & (1-\psi L)
\end{array}\right)
$$

and:

$$
B=\left(\begin{array}{c}
0_{(4 \times 1)} \\
1
\end{array}\right)
$$


where $A(L)$ can be written as $[I-A L]$. Then $w_{t}$ has a $\operatorname{VAR}(1)$ structure: $[I-A L] w_{t}=$ $B \epsilon_{t}$. Obviously, the coefficients of the $A$ and $B$ matrices depend on the deep parameters $\rho, \beta, \delta, \eta$, the parameter $\psi$ governing technology and the steady state value of the level of productivity.

The vector of the state variables is $F_{t}=\left[\begin{array}{ll}k_{t-1} & z_{t}\end{array}\right]^{\prime}$ and:

$$
F_{t}=H F_{t-1}+K \epsilon_{t} \text {. }
$$

Notice that the number of state variables is less than the dimension of the model and it is equal to two. This also implies that the rank $r$ of $\Gamma_{w}(0)$, the static rank, is equal to 2. This model therefore has both dynamic and static reduced rank. Notice also that, for this example, the rank of $A$ is equal to 2 so that the static rank is the same as the rank of the autoregressive lagged matrix therefore implying that the model has common features.

\section{Business Cycle Empirics}

What is the best estimation procedure to recover the dynamic structure of the model economy? We will here compare two alternative strategies. The first is VAR analysis and consists in estimating a reduced form autoregressive model on $w_{t}$, identifying the exogenous shocks using a minimal set of (just-identifying) restrictions and then matching the resulting impulse response functions with the theoretical ones (for a survey of this line of research applied to the study of the effects of monetary policy shocks, see Christiano, Eichenbaum and Evans, 1999). The second exploits explicitely stochastic rank reduction and consists in the estimation of a dynamic factor model. This strategy was first advocated in the macroeconomic literature by Sargent and Sims, 1977 and used for structural analysis by Altug, 1989 and Sargent, 1989. That literature, however, while showing how to test for restrictions on the covariances of the data, did not go as far as showing how to estimate impulse response functions and identify shocks as in VARs. This is why factor models have not been popular tools for empirical structural and policy analysis. In what follows, we show how to identify (common) shocks and impulse response functions in factor models and compare the estimates with those based on VARs.

\subsection{VAR Analysis}

For VAR estimation to be feasible, we must have full static rank since the estimation of $A$ requires the inversion of $\Gamma_{w}(0)$.

As we have seen, the simple model, but this is true for a wide class of models, has reduced static rank and so has the VAR. In the case of the basic model $\Gamma_{w}(0)$ has rank 2 , so that a 5 dimensional VAR cannot be estimated, as $\Gamma_{w}(0)$ cannot be inverted. Reduced static rank might be a characteristic of the theory, but it never occurs empirically, most likely because presence of measurement error in the data "fuzzifies" collinear relations.

With static rank reduction and measurement error, we can estimate a VAR for a block of variables of dimension $r$ provided that the VAR representation for that block 
exists. Alternatively, we can introduce measurement error, and estimate a VAR on the whole system. Let us now analyse the two cases.

\section{A. No measurement Error}

When variables are cleaned from measurement error, estimation can be performed on a block of $w_{t}$, call it $w_{t}^{B}$, so as to obtain a full rank covariance matrix of the variables in the block $\Gamma_{w}^{B}(0)$.

Let us analyse this case for the general model and call the dimension of the block $N_{B}$. It is easily seen that any block has a VMA representation:

$$
w_{t}^{B}=\Theta^{B}(L) \epsilon_{t}
$$

For example, if only the non predetermined variables are included in the block, then:

$$
\Theta^{B}(L)=\left[\Lambda_{1}^{B}(L) C(L)^{-1} D(L)+\Lambda_{2}^{B}(L)\right] \Psi(L)^{-1}
$$

For a VAR representation to exist, the following condition must hold.

Fundamentalness Condition. There exists a $q \times N_{B}$ matrix of filters $\alpha(L)$ in nonnegative powers of $L$ such that:

$$
\alpha(L) \Theta^{B}(L)=I_{q}
$$

This point has been made by Hansen and Sargent, 1990 and Lippi and Reichlin, 1993. For further insight into this issue, see Forni, Lippi and Reichlin, 2002.

If $p_{x}=1, p_{s}=0$ and the exogenous process is fundamental, this condition is satisfied. Hence, for our simple model the condition holds.

If the fundamentalness condition is satisfied, we can approximate the VMA representation with a finite order VAR:

$$
A^{B}(L) w_{t}^{B}=v_{t}^{B}
$$

where $A^{B}(L)$ is a finite order $N_{B} \times N_{B}$ matrix of filters and $v_{t}^{B}=B^{B} \epsilon_{t}$, with $B^{B}$ being a $N_{B} \times q$ matrix $^{2}$.

Notice that $B^{B}$ is an orthonormal rotation of the first $q$ principal component of $\Gamma_{v^{B}}(0)$. Defining as $V$ the $q \times q$ matrix containing its first $q$ eigenvalues and as $J$ the $N_{B} \times q$ matrix of the corresponding eigenvectors, we have: $\epsilon_{t}=R^{\prime} J^{-1 / 2} V^{\prime} v_{t}^{B}$, $\Gamma_{v_{B}}(0)=V J V^{\prime}=B^{B} B^{B^{\prime}}, B^{B}=V J^{1 / 2} R$ where $R R^{\prime}=I_{q}$.

The impulse response function are hence given by:

$$
w_{t}^{B}=A^{B}(L)^{-1} V J^{1 / 2} R \epsilon_{t}
$$

Notice that once we have consistent estimates of $A^{B}(L)$, the impulse response functions can be consistently estimated since the eigenvalues and the eigenvectors are continuous functions of of the matrix entries.

\footnotetext{
${ }^{2}$ Due to the approximation, $\mathrm{E} v_{t}^{B} v_{t}^{B^{\prime}}=\Gamma_{v}(0)$ is not exactly of reduced rank.
} 
An important remark is that the dimension of the rotation matrix, and hence the degree of indeterminacy due to observational equivalence of alternative structures, depends only on the dimension $q$ of the vector of exogenous shocks and not on the dimension of the subsystem $N_{B}$.

\section{B. Measurement Error.}

If the variables have independent measurement error, collinearity disappears and the estimation of the full system is always possible.

Let us assume that measurement error comes in its simplest form, i.e. as a white noise process $\xi_{t} \sim W N\left(0, \Gamma_{\xi}(0)\right)$ orthogonal to the vector of the variables of interest $w_{t}$. Let us refer to the simple model. The vector of measured variables is:

$$
\tilde{w}_{t}=w_{t}+\xi_{t} .
$$

A $V A R(p)$ for $w_{t}$ implies the following $\operatorname{VARMA}(p, p)$ model for the measured equation:

$$
A(L) \tilde{w}_{t}=u_{t}+A(L) \xi_{t}
$$

where $u_{t}=B \epsilon_{t}$.

Standard results indicate that, since the error term is autocorrelated, $A(L)$ and $B$ are not identified and cannot be estimated consistently by a $V A R(p)$.

Two alternative strategies are feasible in this situation. The first is to impose the restrictions and to estimate a factor model. This is what we will analyse in the next Section. The second is to estimate the model by a VAR of order $\tilde{p}>p$ to approximate the VARMA. Let us analyse this strategy here.

Consider the Wold representation of the measured process:

$$
\tilde{w}_{t}=\tilde{\Theta}(L) \tilde{\epsilon}_{t}
$$

where $\tilde{\epsilon}_{t} \sim W . N .\left(0, \Sigma_{\tilde{\epsilon}}\right)$. The impulse response parameters are given by $\tilde{\Theta}(L) \Sigma_{\tilde{\epsilon}}^{1 / 2} \tilde{R}$ with $\tilde{R} \tilde{R}^{\prime}=I_{N_{B}}{ }^{3}$.

The parameters $\tilde{\Theta}(L)$ and $\Sigma_{\tilde{\epsilon}}$ are related to those of the uncontaminated process (2.1) and the measurement error by the spectral identity:

$$
\tilde{\Theta}\left(e^{-i \lambda}\right) \Sigma_{\tilde{\epsilon}} \tilde{\Theta}\left(e^{i \lambda}\right)^{\prime}=A\left(e^{-i \lambda}\right)^{-1} B \Sigma_{\epsilon} B^{\prime} A\left(e^{i \lambda}\right)^{\prime-1}+\Gamma_{\xi}(0)
$$

Notice that, although $\tilde{\Theta}(L)$ can be consistently estimated, one cannot recover $A(L)^{-1} B \Sigma_{\epsilon}^{1 / 2}$ without recovering $\Gamma_{\xi}(0)$. Hence, there exists no rotation matrix $\tilde{R}$

\footnotetext{
${ }^{3}$ Notice that the rotation matrix $\tilde{R}$ is of dimension $N_{B}$. The reason is that, because of the presence of measurement error, $\Gamma_{\tilde{\epsilon}}(0)$ is of full rank.
} 
for which one of the structural shocks has the same impulse response function of the "true" ones. It is interesting to stress that this problem is deeper than the typical identification indeterminacy that pervades the VAR literature (see Christiano, Eichnbaum and Evans, 1999). Even if the researcher knew perfectly the economic model and knew how to choose the appropriate rotation matrix for shocks identification, the presence of measurement error will make inference impossible.

Identity (3.6) shows how measurement errors contaminate the impulse responses parameters of the economic model over the frequency domain. In our example, since the spectral density of the error is constant over $\lambda$ (white noise error), the spectral density of the measured data is close to the spectral density of the model at the frequencies where the variance of the non-contaminated process is high. Hence, if the variance of the process is concentrated in the long-run and at business-cycle frequencies, as in our model economy, then the longer the horizon the closer the impulse response coefficients of the measured data to those of the economic model.

This helps to explain why, empirically, comovement is more clearly detected at longrun frequencies. This feature is also reinforced when the variance of the noise is smaller in the long-run, which is likely to be true empirically.

The empirical performances of the VAR, on the other hand, depend on how well a $\operatorname{VAR}(\tilde{p})$ approximates the $\operatorname{VARMA}(p, p)$ for a given sample size which, in turn, depends on the roots of $A(L)$ and on the size of $\Gamma_{\epsilon}(0)$. The more persistent is the VAR and the larger is $\Gamma_{\epsilon}(0)$, the poorer is the approximation.

\subsection{Factor model estimation}

As Altug, 1989 and Sargent, 1989 have observed, if we add measurement error, the model economy has a factor analytic structure.

The dynamic state space representation for the general case becomes:

$$
\tilde{w}_{t}=A(L)^{-1} B \epsilon_{t}+\xi_{t}=\Theta(L) \epsilon_{t}+\xi_{t}
$$

where $\epsilon_{t}$ is a vector of common shocks of dimension $q$ and $\xi_{t}$ is an idiosyncratic process of dimension $N$ (see, for example, Sargent and Sims, 1977).

When the dynamic lag structure is finite, we can write the model in static form, by stacking lagged variables and we obtain ${ }^{4}$ :

$$
\begin{aligned}
\tilde{w}_{t} & =\Lambda F_{t}+\xi_{t} \\
H(L) F_{t} & =K \epsilon_{t}
\end{aligned}
$$

where $\Lambda$ is a $N \times r$ matrix, $F_{t}$ is $r \times 1$ and $\Lambda F_{t}$ represents the "common component" of $\tilde{w}_{t}$, of dimension $r$, while $\xi_{t}$ is the "idiosyncratic component" of dimension $N$. The impulse response functions are defined, up to a rotation of order $q$ as: $\Theta(L)=\Lambda H(L)^{-1} K \Sigma_{\epsilon}^{-1 / 2}=A(L)^{-1} B \Sigma_{\epsilon}^{-1 / 2}$. The model written in this way, is the static state representation discussed earlier. It can be shown that in the case which

\footnotetext{
${ }^{4}$ For a discussion of the difference between a static and a dynamic factor representation, see Forni, Lippi and Reichlin, 2002
} 
the order of the AR process for $s_{t}$ is less or equal than $p_{s}$, the filter $H(L)$ in $(3.7)$ is of order 1 so that the states have an $\mathrm{AR}(1)$ representation (on this point, see Giannone, Reichlin and Sala, 2002).

In order to perform maximum likelihood estimation, the likelihood of the model can be evaluated using recursive methods (Kalman filtering) or using frequency domain approximations (Sargent, 1989). These methods are computationally expensive and create problems of convergence when the dynamic of the system is very persistent. We propose a less efficient but computationally convenient, two-step procedure. In the first step, the factor loadings $\Lambda$ are estimated as if data were not autocorrelated. The results are then used to estimate the autoregressive paramenters $A(L)$.

Let us analyse the estimation of this model for the basic model example where $H(L)=I-H L, A(L)=I-A L$ and $\xi_{t} \sim W N\left(0, \Gamma_{\xi}(0)\right.$ ) (the results can be easily generalized).

Under the orthogonal measurement error assumption, $\Gamma_{\xi}(0)$ diagonal, the model is identified once we impose the normalization condition $\mathrm{E} F F^{\prime}=I$. The covariances of the variables of interest and of measured data are:

$$
\begin{gathered}
\Gamma_{\tilde{w}}(0)=\Gamma_{w}(0)+\Gamma_{\xi}(0) \\
\Gamma_{w}(0)=\Lambda \Lambda^{\prime} \\
\Gamma_{w}(1)=\Gamma_{\tilde{w}}(1)
\end{gathered}
$$

We will now show how we can recover the impulse response functions $(I-A L)^{-1} B$ from the covariances.

From equation (3.5) and the Yule-Walker equations we have:

$$
\Gamma_{w}(1)=A \Gamma_{w}(0)=A \Lambda \Lambda^{\prime}
$$

Moreover:

$$
\Gamma_{u}(0)=\Gamma_{w}(0)-A \Gamma_{w}(1) .
$$

Our problem is to estimate the parameters of $A$ and $B$ which can be expressed in terms of the factor loadings $\Lambda$ 's. We have:

$$
A=\Lambda H\left(\Lambda^{\prime} \Lambda\right)^{-1} \Lambda^{\prime}, \quad B=\Lambda K
$$

To estimate $A$ we will have to compute the generalized inverse of $\Gamma_{w}(0)$, i.e.:

$$
\Gamma_{w}(0)^{-1}=\left[\Lambda \Lambda^{\prime}\right]^{+}=\Lambda\left(\Lambda^{\prime} \Lambda\right)^{-2} \Lambda^{\prime} .
$$

The estimator for $A$ is:

$$
\hat{A}=\hat{\Gamma}_{w}(1)\left[\hat{\Gamma}_{w}(0)\right]^{+} .
$$

To obtain a consistent estimate of $A$ we need a consistent estimate of $\Lambda$. We use the procedure recently proposed by Doz-Lenglart, 1999. The method is a quasi maximum 
likelihood procedure where the likelihood is first defined as if both the factors and the idiosyncratic components were not autocorrelated and then it is shown that in a stationary framework such estimates are still consistent (see appendix for details).

Once the $\Lambda$ 's are estimated consistently, consistency of $A$ follows from consistency of $\hat{\Gamma}_{w}(1)$. We have:

$$
\operatorname{plim} \hat{A}=\Lambda H\left(\Lambda^{\prime} \Lambda\right)^{-1} \Lambda^{\prime}
$$

We obtain $B$ as an orthonormal rotation of the first $q$ principal component of $\Gamma_{u}(0)$. Defining as $M$ the $q \times q$ diagonal matrix containing the $q$ largest eigenvalues of $\Gamma_{u}(0)$ and as $P$ the $N_{B} \times q$ matrix of the corresponding eigenvectors, we have: $\Gamma_{u}(0)=P M P^{\prime}=B B^{\prime}$ and $B=P M^{1 / 2} R$ where $R R^{\prime}=I_{q}$. The consistency of the empirical counterpart, $\hat{B}$, is a consequence of consistency of $\hat{A}, \hat{\Gamma}_{w}(0)$ and $\hat{\Gamma}_{w}(1)$ since $\Gamma_{u}(0)=\Gamma_{w}(0)-A \Gamma_{w}(1)$.

\section{Empirical comparison}

The exercise here is as follows. We generate the model economy and then estimate it using the VAR procedure and the factor model procedure with and without measurement error. We generate 500 vector time-series $w_{t}=\left(c_{t}, r_{t}, y_{t}, k_{t}, z_{t}\right)^{\prime}$ for our model economy with a sample size $T=200$.

We compute impulse response functions for alternative estimation procedures and report bias, mean squared errors and confidence bands.

The particular model economy is the simple business cycle model where we use the same calibrated parameters as in Uhlig, 1998. They are reported in the Table below.

Table 1. Calibrated Parameters

\begin{tabular}{|l|l|}
\hline$\beta$ & .99 \\
\hline$\rho$ & .36 \\
\hline$\eta$ & 1 \\
\hline$\delta$ & .025 \\
\hline$\psi$ & .95 \\
\hline
\end{tabular}


In Figure 1 we show the sample paths of the five variables for one simulation of the model.

\section{Figure 1. Simulated Path}

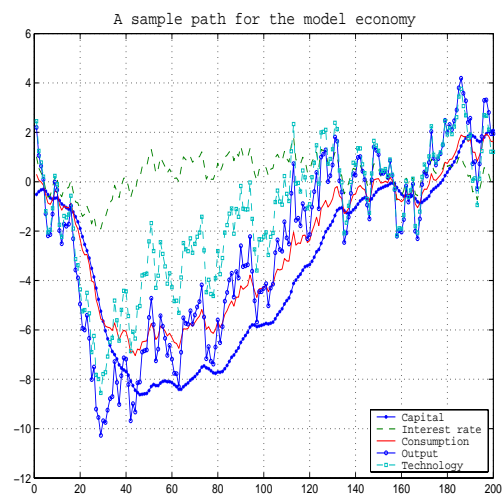

In Figure 2 we show the theoretical impulse response functions in response to a unitary technology shock generated by our model.

\section{Figure 2. Theoretical Impulse Response Functions}

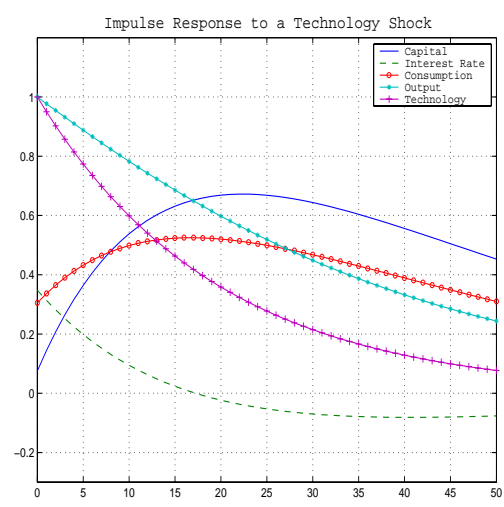

The measurement error is generated as:

$$
\xi_{t} \sim \text { i.i.d.N }\left(\mathbf{0}_{5}, \operatorname{diag}\left[\gamma_{r}, \gamma_{c}, \gamma_{y}, \gamma_{k}, \gamma_{z}\right]\right)
$$

with the $\gamma_{i}$ 's calibrated so that the degree of commonality, given by the ratio $\frac{\operatorname{Var}\left(\tilde{w}_{t}^{i}\right)}{\operatorname{Var}\left(w_{t}^{i}\right)}=1-\frac{\gamma_{i}}{\operatorname{Var}\left(w_{t}^{i}\right)}$, is the same for $i=r, \ldots, z$ and is equal to: $\operatorname{VR}=[1, .9, .8, .6]$.

\section{A. Estimation}

As a full-size VAR on $w_{t}$ cannot be estimated without introducing measurement error, we concentrate on the sub-block $w_{t}^{y c}=\left(y_{t}, c_{t}\right)^{\prime}$. 
We estimate the VAR without measurement error on the sub-block $w_{t}$ by assuming to know the lag length (in this case, 1). Given that we have just one shock there are no identification problems in absence of measurement errors.

With measurement error, the model is a VARMA process with two shocks and the latter must be identified structurally.

For every simulation, we estimate the VAR with a lag length of $p=1, \ldots, 10$ and for every lag length we identify one shock, by choosing one column of the orthonormal rotation matrix $\tilde{R}$ :

$$
\tilde{R}=\left(\begin{array}{cc}
\cos (\theta) & -\sin (\theta) \\
\sin (\theta) & \cos (\theta)
\end{array}\right), \quad \theta \in[-\pi, \pi] .
$$

so as to minimize the sum of the Euclidean distances between the true and the estimated impulse responses for 10 period after the shock for both $y_{t}$ and $c_{t}$.

For every $V R$ we obtain 500 impulse responses for each lag length $p=1, \ldots, 10$.

We choose the optimal lag length $p^{*}$ as the one that gives the minimum MSE for 10 periods after the shock. It turns out that for every $V R$ the optimal lag length is 7 .

For the estimation using factor analysis, we assume here that $r$ and $q$ are known, as we have done for the number of lags in VAR estimates. Notice, however, that since we are using a likelihood procedure, we could have used the test for the number of common factors proposed by Doz and Lenglart, 1999.

\section{B. Comparison}

A comparison between the two alternative methods is provided in what follows.

For each value of $V R$ we report 4 figures.

These figures display, respectively, the bias, the mean squared error ${ }^{5}$ and the confidence bands ${ }^{6}$ for the estimates of the impulse response functions for the two variables under scrutiny, $y_{t}$ and $c_{t}$ and for both the VAR and the factor model, taking as a benchmark the bivariate VAR estimation without measurement error.

For example, Figures 3 and 4 show the bias and the MSE of the estimated impulse response functions in the case $V R=.9$ for $c_{t}$ (top) and $y_{t}$ (bottom).

The statistics for the "clean" VAR, the starred lines, are reported for comparison.

Figures 5 and 6 report the confidence bands computed from, respectively, a VAR on $w_{t}^{y c}$ (without measurement error), a VAR on $\tilde{w}_{t}^{y c}$ (with measurement error) and the factor model (from left to right). The true impulse response functions are reported for comparison (bold line).

\footnotetext{
${ }^{5}$ The statistics for the bias and the MSE are the means of the empirical distributions

${ }^{6} 95 \%$ confidence bands are computed from the empirical distribution function by taking the 2.5-th and the 97.5 -th percentile.
} 
Figure 3. Bias - VR $=0.9$
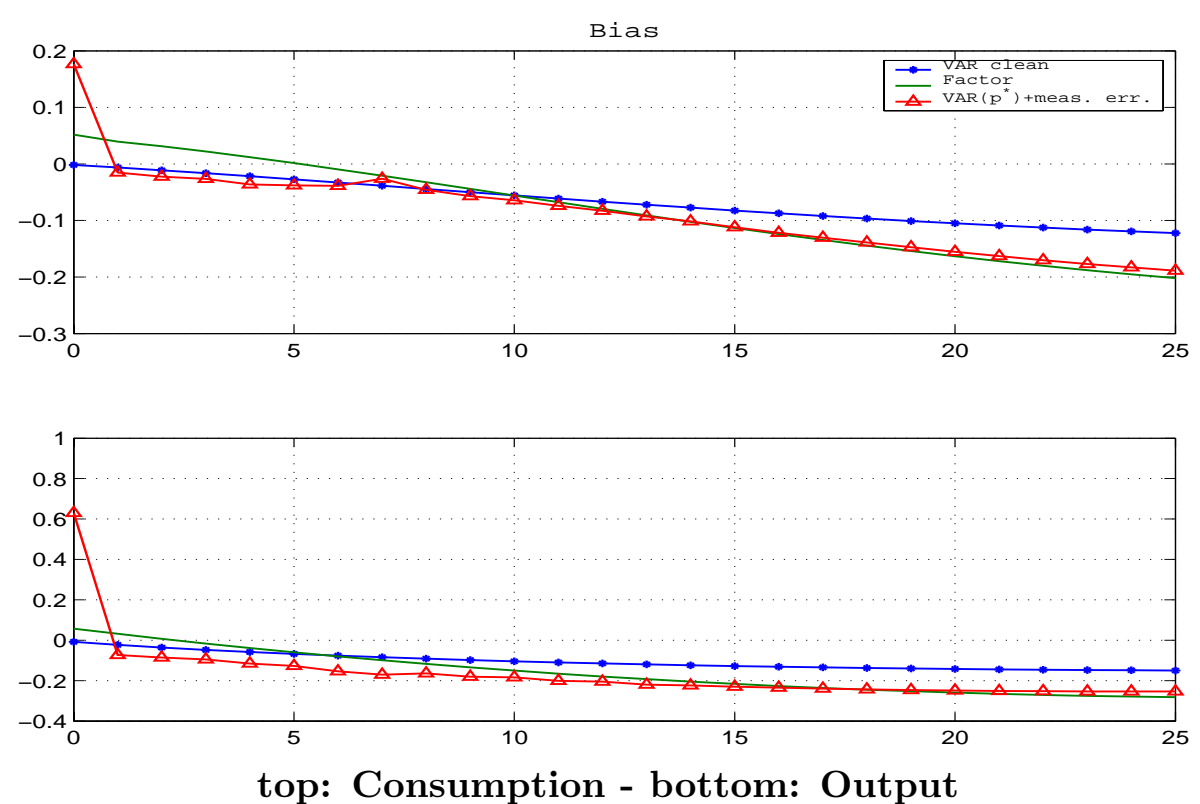

Figure 4. Mean Squared Error - VR $=0.9$
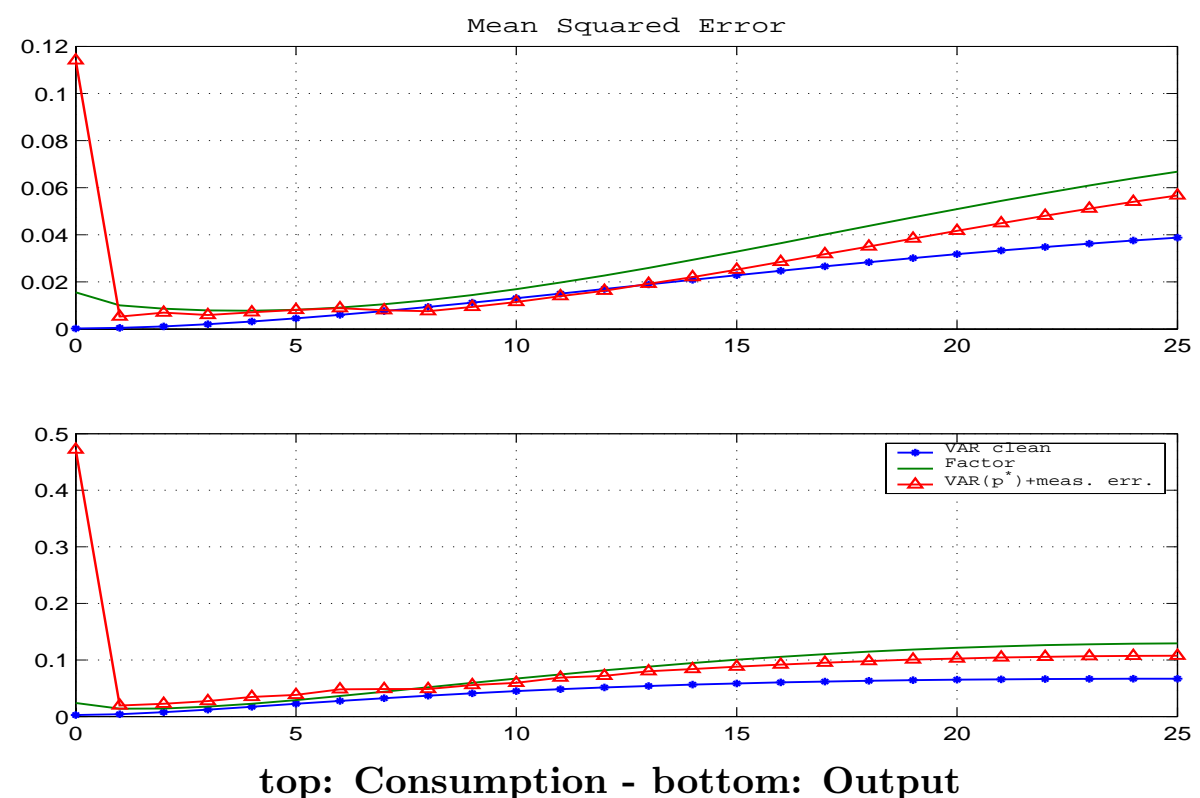
Figure 5. Comparison of Impulse Response Functions - VR $=0.9$
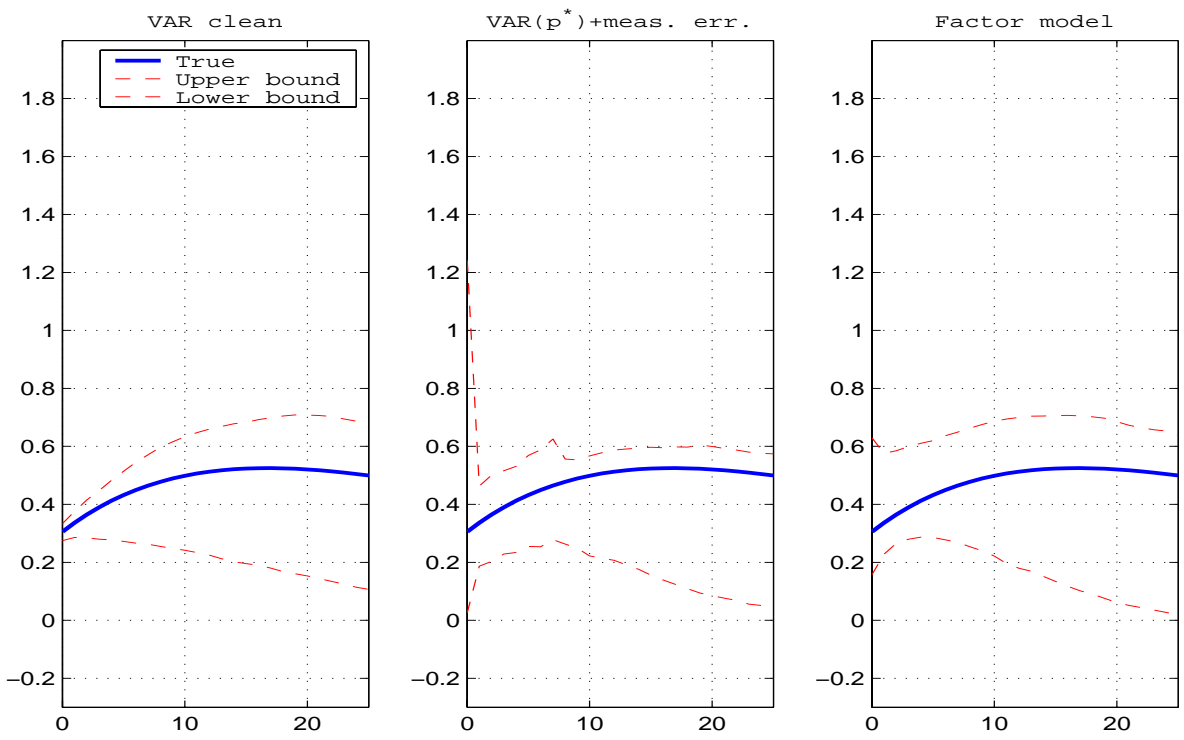

Consumption

Figure 7. Comparison of Impulse Response Functions - VR $=0.9$
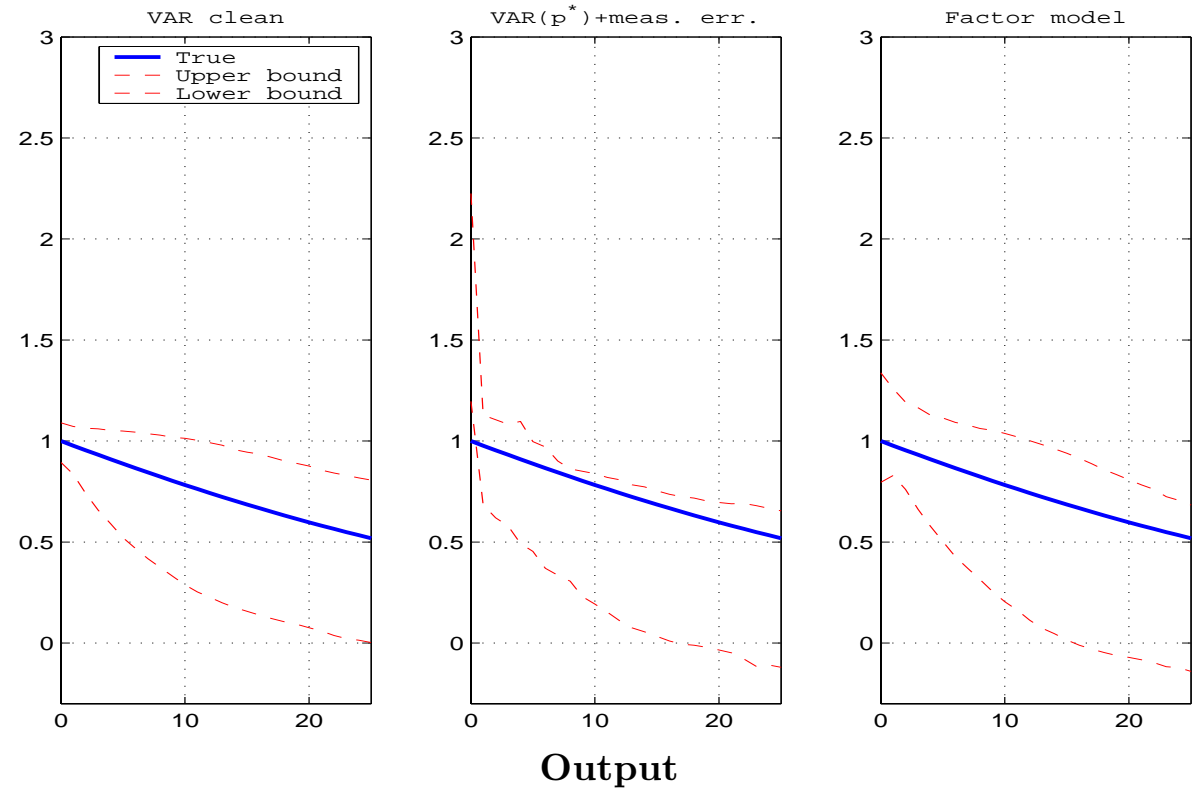
Figure 8. Bias - VR $=0.8$
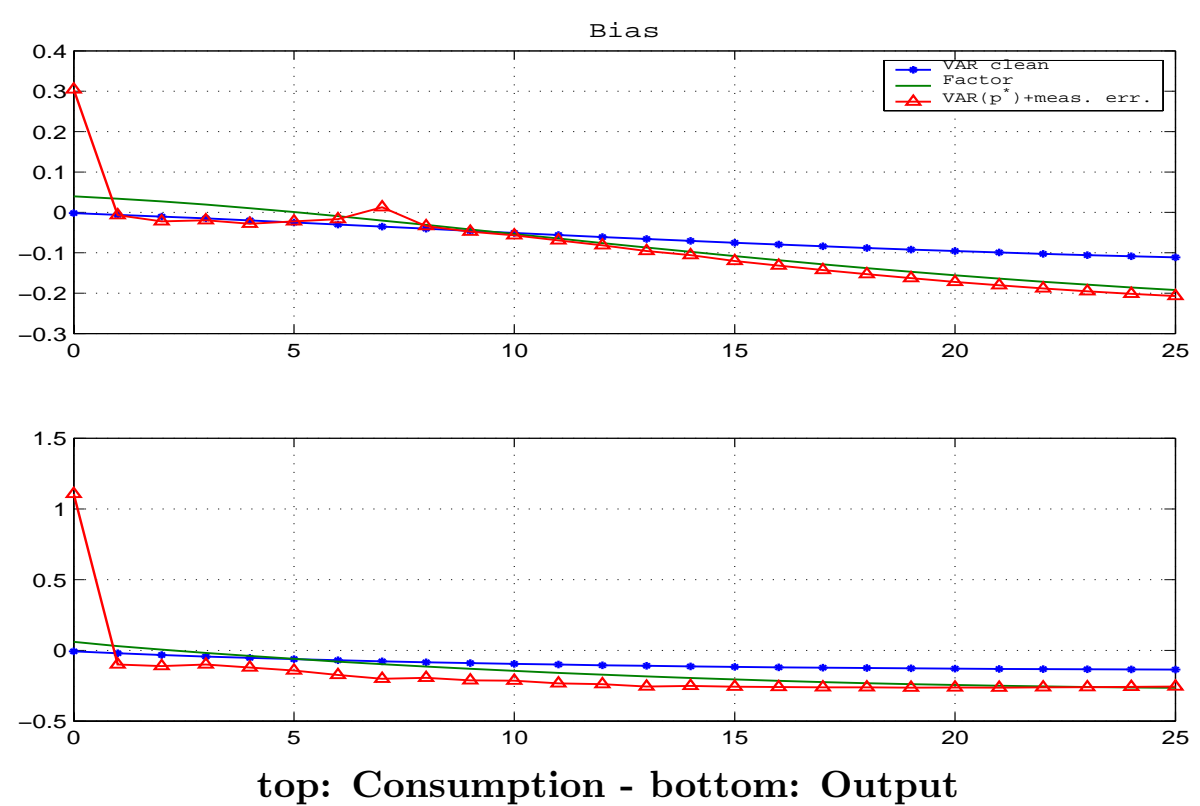

Figure 9. Mean Squared Error - VR $=0.8$
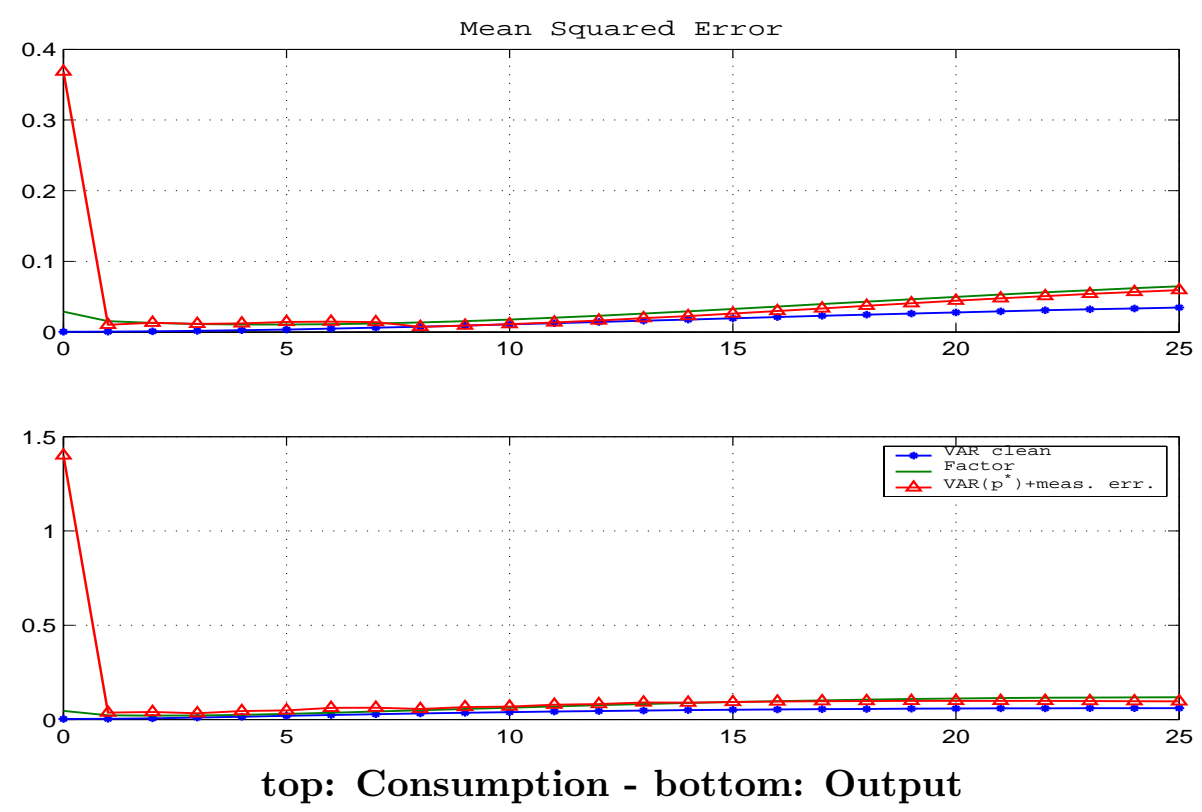
Figure 10. Comparison of Impulse Response Functions - VR $=0.8$
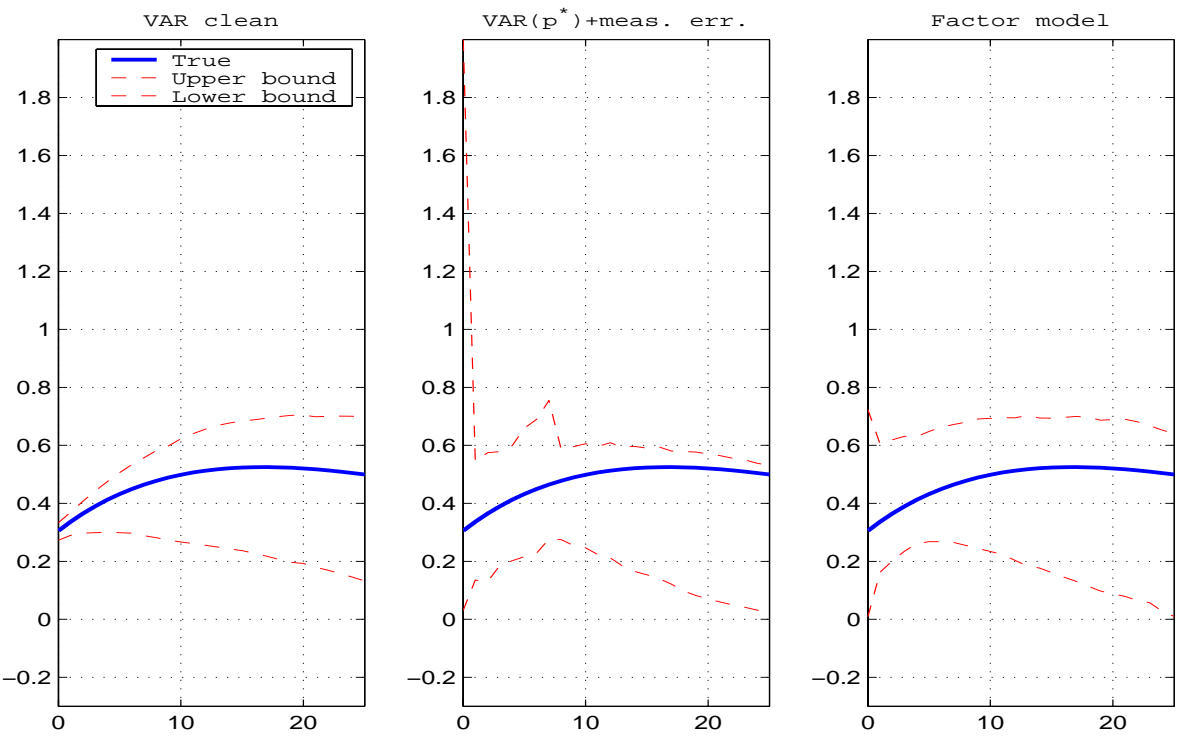

Consumption

Figure 11. Comparison of Impulse Response Functions - VR $=0.8$
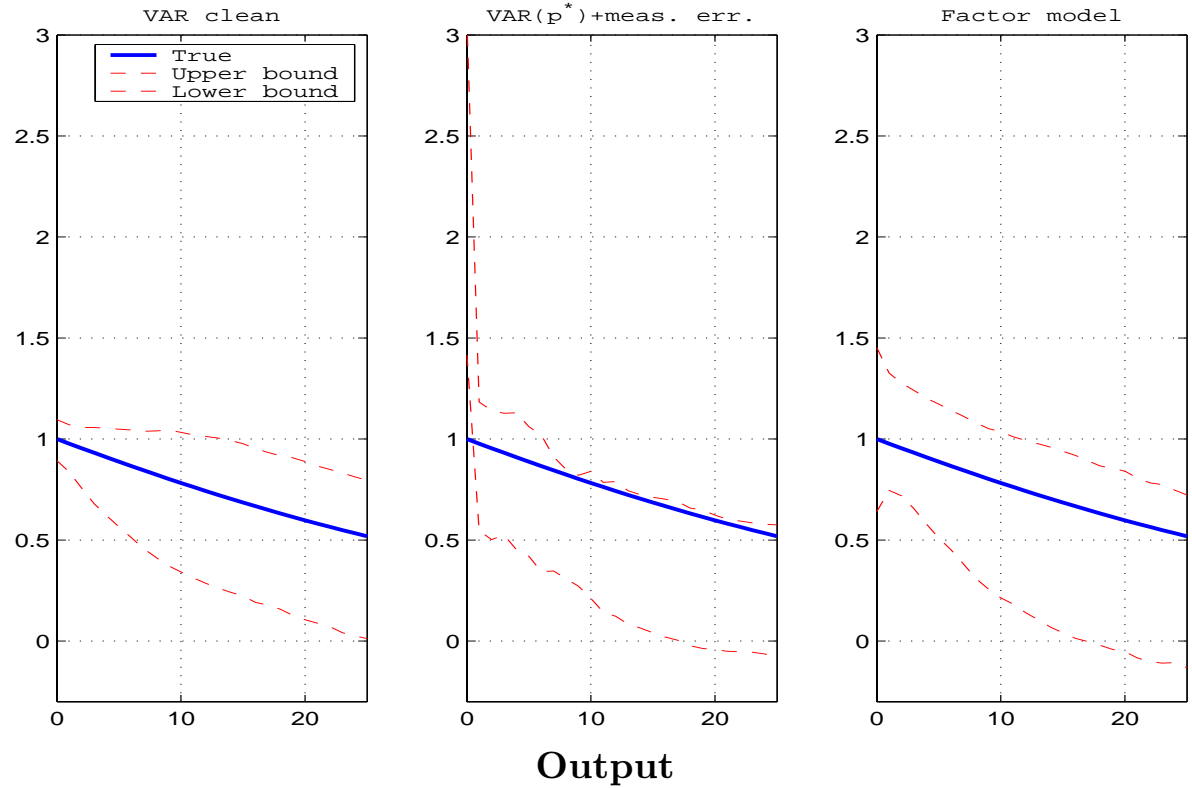
Figure 12. Bias - VR $=0.6$
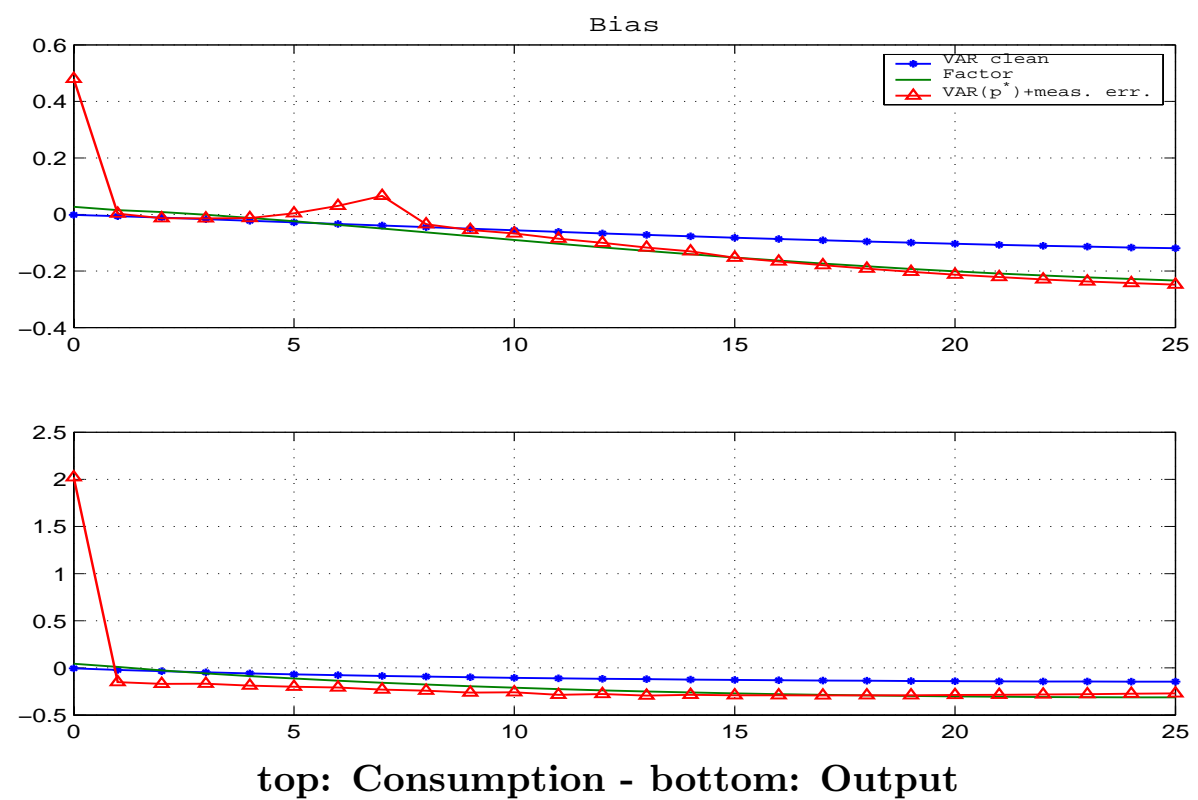

Figure 13. Mean Squared Error - VR $=0.6$
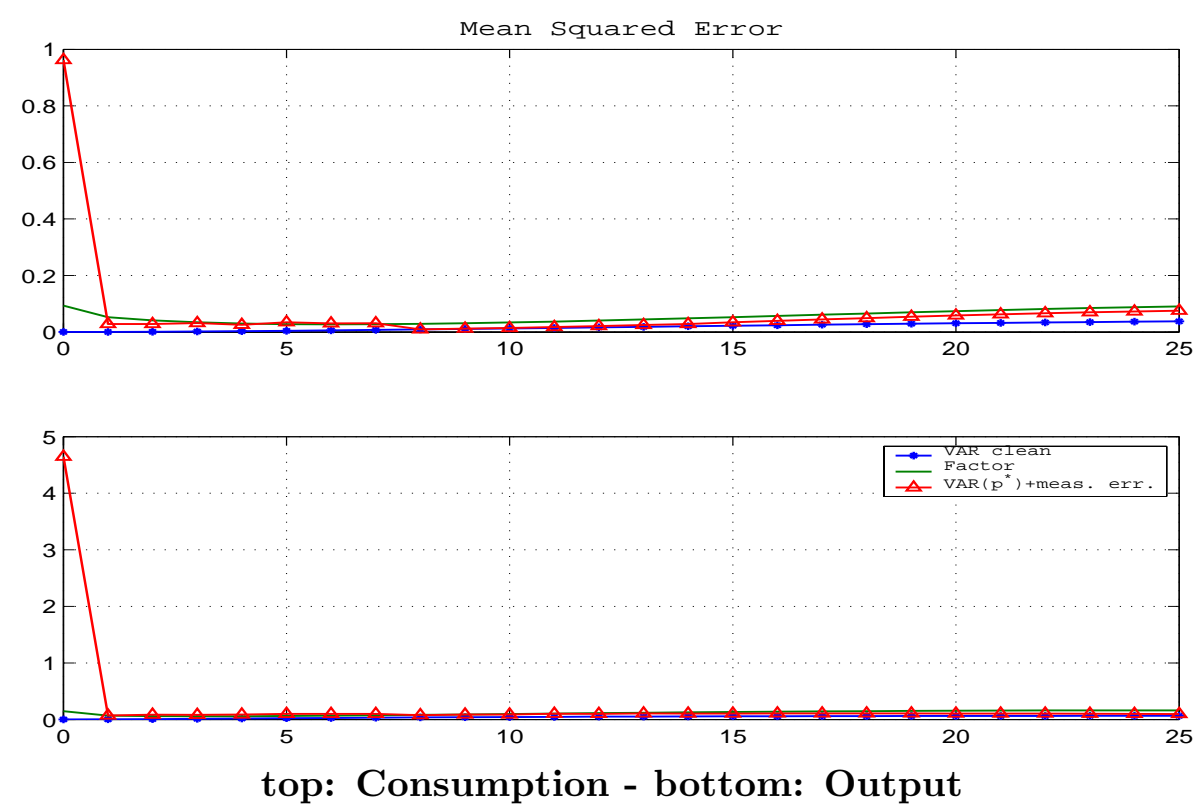
Figure 14. Comparison of Impulse Response Functions - VR $=0.6$
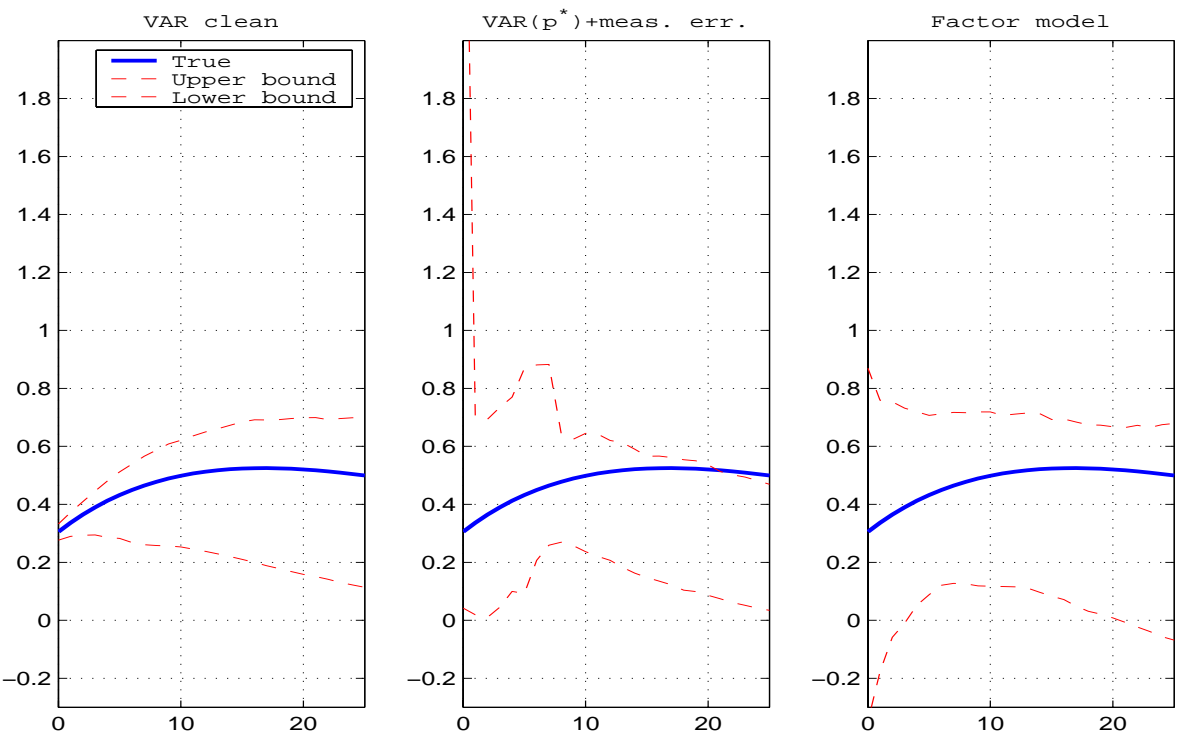

Consumption

Figure 15. Comparison of Impulse Response Functions - VR $=0.6$
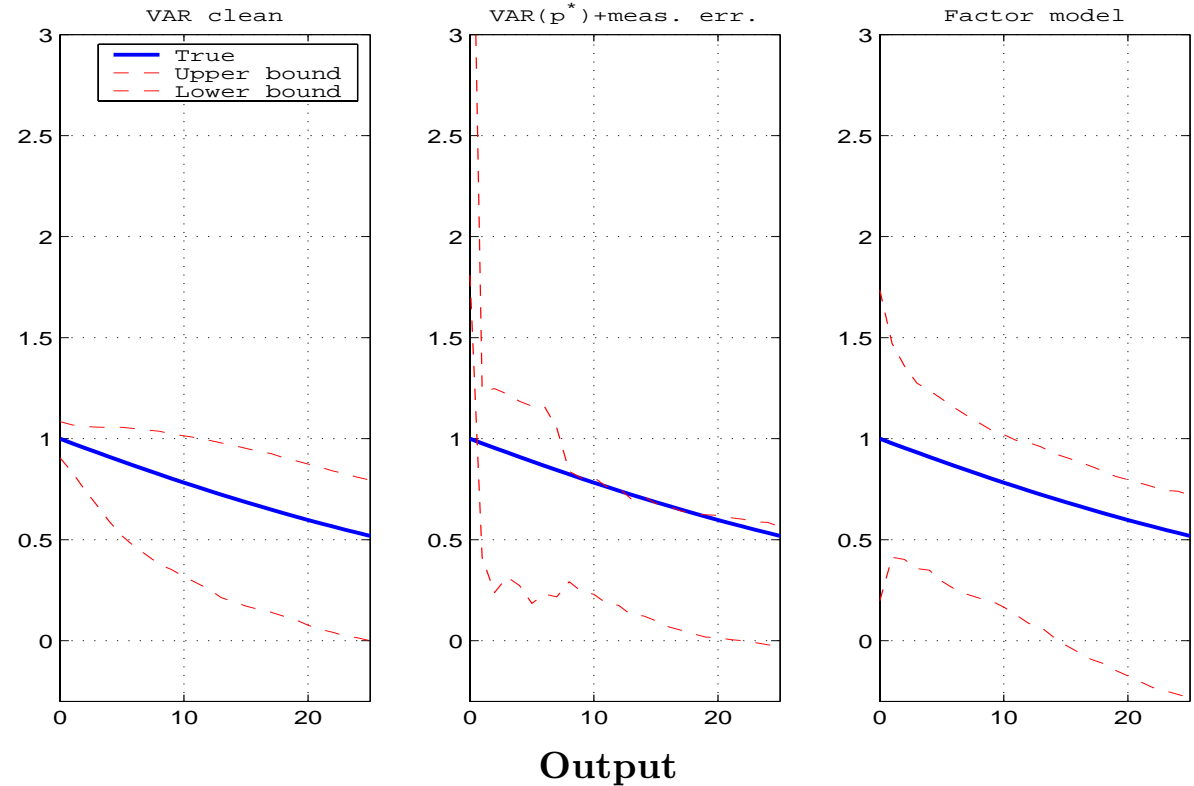

We highlight the following features.

First, and this demonstrates empirically the results of the previous Section, even a small measurement error $(V R=.9)$ is sufficient to spoil the inference drawn from the VAR on short-run impulse response coefficients. It is evident that the VAR does not consistently estimate the true contemporaneous response of the system (see for example Figures from 3 to 6 ). 
Second, in the case of the "contaminated" VAR, as measurement error increases (VR decreases), both the bias and the mean squared error at short time horizons become larger. This results suggest that impulse response coefficients estimated using VAR are more reliable at medium and long run horizon.

As $V R$ becomes smaller, the factor model has harder and harder times in estimating efficiently the impulse responses, as one can see from the fact that confidence bands widen ${ }^{7}$. However, factor estimates of impulse response functions are more accurate than VAR estimates at short horizons while the two methods perform similarly at medium and long run horizons.

\section{5 large: Discussion}

As mentioned in the introduction, factor models have recently gained popularity as a parsimonios method to estimate dynamic relations in large panels of time series. This recent literature advocates principal component methods for the estimation of the factor space and provide consistency results and rates as the dimension $n$ of the cross section and the time dimension $T$ go to infinity. The model analysed in this literature are more flexible than standard factor models since the idiosyncratic component is typically allowed to contain cross-correlated elements. Under these conditions, common and idiosyncratic components are not identified for $n$ fixed and this makes it impossible to use maximum likelihood estimation; higher flexibility in the parametrization of the model comes at a cost of possible loss of efficiency. Comparing finite sample estimation performance for this class of econometric models and the likelihood based ones is an interesting project, but not the one pursued here. Let us here instead make few remarks on what we would gain in adopting the large cross section approach, instead of the $n$ fixed approach used in this paper, for the purpose of the structural analysis we have discussed.

First of all, let us oberve that the model with $n$ large corresponds to a model with a large number of states such as, for example, the model with heterogenous capital (Campbell, 1997) or models with heterogenous agents and different information sets. These models are therefore more complex than the benchmark business cycle example analysed here, but they belong to the same family. From the econometric point of view, information contained in a large cross-section helps in cleaning from measurement error. The intuition is that principal components are linear combinations of the measured variables of the panel and they become increasingly collinear with the underlying variables of interest as $n$ increases (the idiosyncratic component capturing measurement error dies on average by a law of large number mechanism). To obtain consistent estimates of the impulses, we just need consistent estimates of the factor loadings and the shocks are identified as rotations of principal components of the residuals of the VAR on the factors (see Giannone, Reichlin and Sala, 2002 and Forni, Lippi and Reichlin,

\footnotetext{
${ }^{7}$ We could have used other methods to estimate the factor model (for example, the Kalman filter). We want to point out two things. First, our estimated parameters can be thought as consistent preliminary estimates to be used as starting values for the Kalman recursion. The two-step method used here provides anyway accurate results. Second, we did some experiments with the Kalman filter, but the convergence was very sensitive to the choice of the starting values
} 
2002). As $n$ increases, the number of shocks to identify remains fixed at $q$. No matters how large is the number of states, the complexity of the identification problem depends on the number of exogenous shocks $q$.

\section{Summary and conclusions}

Both theory and empirics suggest that there are fewer macroeconomic shocks than variables. When variables are measured with errors, the measured equations generated by equilibrium business cycle models have a dynamic factor structure and the reduced form follows a VARMA model. We have compared VAR and structural factor estimation as two alternative approximations to these equations using a benchmark business cycle model to generate the data and under alternative assumptions on the size of measurement error. We have shown that, at short horizons, VAR estimates of impulse response functions are less accurate than factor estimates while the two methods perform similarly at medium and long run horizons.

\section{References}

[1] Altissimo, F., Bassanetti, A., Cristadoro, R., Forni, M., Hallin, M., Lippi, M., Reichlin, L., Veronese G.F. (2002), "A real time coincident indicator for the euro area", CEPR discussion paper.

[2] Ahn, S. K. and Reinsel G. C. (1988), "Nested Reduced-Rank autoregressive models for multiple time series", Journal of American Statistical Association, 83, pp. 849856.

[3] Altug, S. (1989), "Time-to-Build and Aggregate Fluctuations: Some New Evidence", International Economic Review, Vol. 30, No. 4, pp. 889-920.

[4] Campbell. J. R. (1997), "Entry, Exit, Embodied Technology and Business Cycles", NBER Working Paper \# 5955.

[5] Christiano. L. J.(2001), "Solving Dynamic Equilibrium Models by a Method of Undetermined Coefficients", mimeo, Northwestern University.

[6] Christiano, L. J., M. Eichenbaum, and C. L. Evans (1999), "Monetary Policy Shocks : What Have We Learned and to What End?", In J. B. Taylor and M. Woodford, Eds., Handbook of Macroeconomics, (North Holland, Amsterdam).

[7] Doz C. and Lenglart F. (1999), "Analyse Factorielle Dynamique: Test du Nombre de Facteurs, Estimation et Application á l'Enquête de Conjoncture Dans l'Industrie", Annales d'Economie et de Statistique, 54, pp. 91-127.

[8] Engle R. F. and Kozicki S. (1993), "Testing for Common Features", Journal of Business and Economic Statistics, 11, pp. 369-395. 
[9] Engle and Watson (1983), "Alternative Algorithms for the Estimation of Dynamic Factor, MIMIC, and Varying Coefficient Regression Models", Journal of Econometrics, 23, pp. 385-400.

[10] Forni M., Lippi, M. and Reichlin, L. (2002), "Opening the Black Box: Identifying Shocks and Propagation Mechanisms in VARs and Factor Models", mimeo.

[11] Forni M., Hallin, M., Lippi, M. and Reichlin, L. (2000), "The Generalized Factor Model: Identification and Estimation", The Review of Economics and Statistics, November.

[12] Forni M. and Reichlin, L. (1998), "Let's Get Real: A Factor Analytical Approach to Disaggregated Business Cycle Dynamics", The Review of Economic Studies, Vol. 65, No. 3, pp. 453-473.

[13] Geweke J. (1977), "The Dynamic Factor Anlysis of Economics Time Series Model", in D. Aigner and A. Goldberger (eds.), Latent Variables in Socioeconomic Models, pp. 365-383. (Amsterdam: North-Holland).

[14] Geweke J. F. and Singleton K. J. (1981), "Maximum Likelihood "Confirmatory" Factor Analysis of Economic Time Series", International Economic Review, Vol. 22, No. 1, pp. 37-54.

[15] Giannone D., Reichlin, L. and Sala, L. (2002), "Tracking Greenspan: Systematic and Unsystematic Monetary Policy Revisited", CEPR Discussion Paper \# 3550.

[16] Hansen, L. P. and Sargent T. J. (1980), "Formulating and Estimating Dynamic Linear Rational Expectations Models“, Journal of Economic Dynamics and Control, 2, 1-46.

[17] Hansen, L. P. and Sargent T. J. (1990), "Two Difficulties in Interpreting Vector Autoregressions", in Hansen and Sargent, Rational Expectations Econometrics, Westview Press, Boulder and London.

[18] Harvey, A.C. (1989), Forecasting Structural Time Series Models and the Kalman Filter, Cambridge University Press.

[19] King, R.G., Plosser C.I. and Rebelo S. T. (1991), "Production, Growth and Business Cycles: I. The Basic Neoclassical Model", Journal of Monetary Economics 21, pp. 195-232.

[20] King, R.G., Stock, J.H., Plosser C.I. and Watson M. (1991), "Stochastic Trends and Economic Fluctuations", The American Economic Review, September, pp. 819-839.

[21] Kydland, F.E. and Prescott, E.C. (1983), "Time to Build and Aggregate Fluctuations", Econometrica, 50, 1345-70. 
[22] Lippi M. and Reichlin L. (1993), "The Dynamic Effects of Aggregate Demand and Supply Disturbances: Comment", The American Economic Review, Vol. 83, No. 3, pp. 644-652.

[23] Long, J.B. Jr. and Plosser, C.I. (1983), "Real Business Cycles", The Journal of Political Economy, 91, 39-69.

[24] Sargent, T. J. (1989), "Two Models of Measurements and the Investment Accelerator"

The Journal of Political Economy, Vol. 97, No. 2, pp. 251-287.

[25] Sargent T. J. and Sims, C. A. (1977), "Business Cycle Modelling Without Pretending to Have Much a Priori Economic Theory", in C. Sims (ed.) New Methods in Business Research (Minneapolis, Federal Reserve Bank of Minneapolis).

[26] Stock J. and Watson M. (1998), "Diffusion Indexes", NBER Working Paper \# 6702 .

[27] Vahid F. and Engle R. F. (1993), "Common Trends and Common Cycles" Journal of Applied Econometrics, Vol. 8, No. 4, pp. 341-360.

[28] Velu R. P., Reinsel G. C. and Wichern D. W. (1986), "Reduced Rank Models for Multiple Time Series"

Biometrika, 73, pp. 105-118.

[29] Watson M. W. (1993), "Measures of Fit for Calibrated Models", The Journal of Political Economy, Vol. 101, No. 6, pp. 1011-1041.

[30] Uhlig H. (1998), "A Toolkit for Analyzing Nonlinear Dynamic Stochastic Models easily", manuscript, CentER, University of Tilburg.

\section{Appendix: Doz-Lenglart procedure}

Doz and Lenglart, 1999, consider the quasi-likelihood of the model (3.7), computed under a Gaussian assumption as if neither the factors nor the idiosyncratic component were autocorrelated. The quasi-likelihood can be written, up to a constant term, as:

$L_{T}\left(w_{1}^{M}, \ldots, w_{T}^{M} ; \Lambda, \Gamma_{\xi}(0)\right)=-\frac{n}{2} \ln \left(\operatorname{det}\left(\Lambda \Lambda^{\prime}+\Gamma_{\xi}(0)\right)\right)-\frac{n}{2} \operatorname{trace}\left(\left(\Lambda \Lambda^{\prime}+\Gamma_{\xi}(0)\right)^{-1} \hat{\Gamma}_{w^{M}}(0)\right)$

The maximum likelihood estimates, $\hat{\Lambda}$ and $\hat{\Gamma}_{\xi}(0)$, are the solution of the following system (cfr. Magnus and Neudecker, 1988):

$$
\Lambda=\hat{\Gamma}_{w^{M}}(0)\left(\Lambda \Lambda^{\prime}+\Gamma_{\xi}(0)\right)^{-1} \Lambda
$$




$$
\Gamma_{\xi}(0)=\operatorname{diag}\left(\hat{\Gamma}_{\mathrm{w}^{\mathrm{M}}}(0)-\Lambda \Lambda^{\prime}\right)
$$

If $\Gamma_{\xi}(0)$ were known, (7.8) would be satisfied if:

$$
\hat{\Lambda}=\left(\Gamma_{\xi}(0)\right)^{1 / 2} V_{r} J_{r}^{1 / 2}
$$

where $J_{r}$ is the $r \times r$ diagonal matrix conaining the $r$ largest eigenvalues of:

$$
\left(\Gamma_{\xi}(0)\right)^{-1 / 2}\left(\hat{\Gamma}_{w^{M}}(0)-\Gamma_{\xi}(0)\right)\left(\Gamma_{\xi}(0)\right)^{-1 / 2}
$$

and $V_{r}$ is the $N \times r$ matrix of corresponding orthonormal eigenvectors.

Thus, (7.10) provides an explicit solution for $\Lambda$ as a function of $\Gamma_{\xi}(0)$ and (7.9) gives $\Gamma_{\xi}(0)$ as an explicit solution of $\Lambda$. The solution of the system (7.8) and (7.9) can hence be found iteratively choosing an appropiate starting value for $\Gamma_{\xi}(0)$.

Doz and Lenglart, 1999, show that if $F_{t}$ and $\xi_{t}$ are weakly stationary, then $\hat{\Lambda}$ and $\hat{\Gamma}_{\xi}(0)$ are consistent:

$$
\operatorname{plim} \hat{\Lambda}=\Lambda, \quad \operatorname{plim} \hat{\Gamma}_{\xi}(0)=\Gamma_{\xi}(0), \text { as } \mathrm{T} \rightarrow \infty
$$

